\title{
Le 18 Brumaire, une étape dans la constitution d'une armée nouvelle
}

\section{Annie Crépin}

\section{(2) OpenEdition \\ Journals}

Édition électronique

URL : https://journals.openedition.org/ahrf/291

DOI : 10.4000/ahrf.291

ISSN : 1952-403X

Éditeur :

Armand Colin, Société des études robespierristes

Édition imprimée

Date de publication : 1 décembre 1999

Pagination : 663-676

ISSN : 0003-4436

Référence électronique

Annie Crépin, «Le 18 Brumaire, une étape dans la constitution d'une armée nouvelle », Annales

historiques de la Révolution française [En ligne], 318 | octobre-décembre 1999, mis en ligne le 11 avril

2006, consulté le 24 avril 2022. URL : http://journals.openedition.org/ahrf/291 ; DOI : https://doi.org/

10.4000/ahrf.291

Ce document a été généré automatiquement le 24 avril 2022.

Tous droits réservés 


\title{
Le 18 Brumaire, une étape dans la constitution d'une armée nouvelle
}

\author{
Annie Crépin
}

1 Napoléon Bonaparte, héritier de la Révolution, transforma bien des créations de celle-ci et du Directoire afin de les plier à ses desseins personnels. Mais il en est une qu'il trouva achevée lors de son arrivée au pouvoir, parce qu'elle était déjà une institutionnalisation des expériences de la Révolution. Ce fut la conscription fondée par la loi Jourdan du 19 fructidor an VI. L'invention d'une armée nouvelle devait tout, en effet, à la période qui précéda le coup d'État. Celui-ci fut même facilité par cette création dont les effets pervers échappaient à ses auteurs.

2 Certes, l'Empereur fit subir à la loi des violations grandissantes au fur et à mesure de son règne au point que, faussée dans son application, l'institution devint odieuse à la population - sans que son impopularité fût générale. Au demeurant, la Restauration qui l'avait d'abord supprimée la rétablit tant elle mesura, ainsi que chacun des régimes qui lui succéda au XIX ${ }^{\mathrm{e}}$ siècle, la force irrésistible de cette armée nouvelle. Force qui était fondée sur des rapports, eux aussi nouveaux, avec une nation, une citoyenneté et une société profondément transformées depuis 1789.

I. Armée et nationLa « mobilisation générale » le peuple, la Nation, l'État

3 La Constituante, qui avait déclaré la paix au monde en 1790, se défiait de la «chose militaire » malgré la réflexion entamée par les «militaires-philosophes» du XVIII ${ }^{\mathrm{e}}$ siècle, tels Guibert et Servan, qui contenait en germe le concept de nation-armée ${ }^{1}$. En décembre 1789, les Constituants avaient repoussé avec véhémence le soldat-citoyen proposé par Dubois-Crancé parce qu'ils redoutaient que, derrière cette figure, ne se profilât une militarisation de la nation, jugée incompatible avec la liberté de ses membres et plutôt associée, dans leur esprit, aux structures d'une société barbare et archaïque ${ }^{2}$. En même temps, ils se méfiaient de l'armée royale composée de ceux qui faisaient métier de la guerre car ils la jugeaient, à tort ${ }^{3}$, coupée de la nation et pensaient qu'elle était l'instrument passif de l'absolutisme. Tout au plus firent-ils des réformes qui tendaient à adapter l'armée au nouveau cours des choses ${ }^{4}$. 
Or, trois ans plus tard, la Convention vote le décret du 23 août 1793 qui n'ordonne pas seulement la levée en masse des jeunes requis mais oblige chacun - et chacune - à contribuer à l'effort de guerre selon ses capacités et sa situation. Dans l'esprit des Montagnards, il ne s'agit pas seulement d'une acceptation des nécessités de la guerre elle avait toujours existé - il s'agit aussi et surtout de l'accomplissement d'un acte patriotique que le caractère, révolutionnaire à tous les sens du terme, de la fabrication du salpêtre symbolise parfaitement. "Que chaque citoyen soit potentiellement un militaire, ceci matérialise un rapport à l'État qui s'appelle la mobilisation générale " ${ }^{5}$. Cette militarisation du peuple, même s'il ne se portait pas en son entier sur le champ de bataille, ne résultait pas seulement de l'aggravation de la situation militaire qui aurait poussé les Conventionnels à faire flèche de tout bois. Le cours des événements avait fait que des valeurs universalistes étaient prises en charge par une nation particulière à laquelle revenait le soin de les défendre les armes à la main ${ }^{6}$. D'où la nécessité de redéfinir les liens entre le peuple, la nation et l'État centralisateur, le patriotisme étant la médiation entre cet État et la nation ou plutôt les valeurs universalistes qu'elle prétendait continuer à incarner.

«La relation que la guerre établit entre l'état et le peuple - la mobilisation générale - permet d'atteindre la forme la plus pure de la volonté générale. C'est la nation en armes...ce n'est pas l'État qui combat. C'est le peuple, source de toute souveraineté. $»^{7}$

5 À la limite, si la défense de la nation devenait l'affaire de tous, ce pouvait être l'antidote à l'avènement d'un chef militaire victorieux que redoutaient certains, tel Robespierre.

De l'armée-nation à l'armée nationale, mythes et réalités

6 Pour autant, la nation-armée qui semblait «surgir » du décret de 1793 ne saurait se confondre avec les mythes qui, plus tard et pendant longtemps, lui furent associés. Il avait fallu trois ans pour passer du volontariat à la réquisition. L'appel aux volontaires avait d'ailleurs été conçu comme un palliatif ou, du moins en 1791, comme le moyen de constituer une force complémentaire et non un substitut de l'armée de ligne. Trois ans représentent un délai relativement long dans un temps révolutionnaire et traduisent les hésitations des assemblées.

7 Cette armée-nation n'était en aucun cas le peuple levé spontanément sans aucune instruction préalable et qui aurait été nécessairement victorieux parce qu'il défendait une cause juste, comme on l'a prétendu après, en forgeant une légende autour de la victoire de Valmy. Il ne s'agissait pas non plus d'une force de résistance, au sens moderne du terme, engagée dans une lutte de partisans. Celle-ci avait pu être menée par certaines communautés rurales pendant un bref laps de temps. Mais ce furent parfois les mêmes qui s'opposèrent à la levée en masse puis à la loi Jourdan ${ }^{8}$.

8 Ce n'est d'ailleurs pas sans réticences que le Comité de salut public et la Convention avaient décrété la levée en masse et encore avaient-ils voulu corriger et encadrer l'élan du peuple de Paris qui rêvait d'une «levée tumultuaire ». La mobilisation de la nation tout entière ne signifiait l'envoi sur le champ de bataille que d'une fraction d'entre d'elle, déterminée par l'âge - les jeunes de 18 à 25 ans - et par la situation de famille les célibataires et les veufs sans enfants. Par ailleurs, dans la pensée des sans-culottes, il s'agissait, par ce mouvement, de se porter au-devant de l'armée pour lui donner un «coup de main » temporaire. C'était déjà la conception des volontaires de 1791 qui abandonnèrent les drapeaux en 1792, malgré les objurgations de la Convention, en 
s'appuyant sur une modalité ambiguë du décret de 1791, leur réservant le droit de se libérer de leur engagement à la fin de la campagne prévue le 1er décembre 1792.

9 S'agissait-il davantage dans la pensée des Montagnards d'un service de longue durée ? L'urgence de la situation avait imprimé son sceau au décret du 23 août: aucune périodicité n'était définie, aucune relève annuelle des plus anciens par les plus jeunes n'était prévue en temps de paix et rien ne disait en fait que le décret serait reconduit si la guerre s'achevait. Les événements devaient trancher puisque la mesure fut considérée comme terroriste et, comme telle, abolie avec le système de l'an II. L'effet pervers de cette suppression fut de faire retomber le fardeau exclusivement sur ceux «...qui avaient eu le malheur d'avoir eu entre 18 et 25 ans en $1793{ }^{9}$. Situation qui fut ressentie comme une injustice sur fond de guerre qui se prolongeait et qui était de moins en moins perçue comme défensive les conséquences en furent une désertion massive dès l'an III mais aussi, pour ceux qui combattaient de plus en plus loin de leur pays et de leur milieu d'origine, le fait qu'ils étaient ainsi coupés de la nation et redevenaient des professionnels.

Les hommes de la Révolution n'avaient certes jamais prétendu supprimer le métier des armes ni abolir ce que l'on appellera plus tard l'armée permanente. Certes, comme leurs devanciers de la Constituante, les Montagnards gardaient une grande méfiance envers le pouvoir militaire ou plutôt envers un pouvoir militaire qui constituerait une force séparée de la nation. Ils poussèrent à l'extrême l'affirmation de la supériorité du pouvoir civil jusqu'à faire tomber la « faux révolutionnaire sur la tête des généraux. Depuis 1791 pour les volontaires, et à partir de 1792 pour l'armée royale, l'organisation du recrutement dépendit des civils élus, jusqu'à l'échelon de la commune. La Convention montagnarde paracheva l'évolution de l'armée de ligne. La réalisation progressive de l'amalgame, dont Dubois-Crancé était l'un des inspirateurs, fut un moyen de conjuguer l'ardeur civique des volontaires et l'expérience professionnelle des soldats de métier.

11 Il faut remarquer que les historiens ont récemment démythifié cette distinction simpliste entre les uns et les autres tant il leur parait difficile d'opposer, même en 1791, le «pur volontaire mu seulement par des considérations patriotiques, et le «vil mercenaire, à tout le moins le professionnel indifférent à l'évolution de la nation pour laquelle il combattait. Outre qu'en 1792, un soldat sur cinq n'était pas encore enrôlé en 1789 et avait donc vécu dans un pays en pleine ébullition, les quatre autres composaient un microcosme de la nation, qui reproduisait les bouleversements de celle-ci.

Mais, quelle que soit l'origine de ces hommes, qu'ils soient destinés à revenir dans leurs foyers ou à rester sous les drapeaux, les Montagnards entendirent qu'ils ne se battent plus pour leurs généraux ou pour un monarque mais pour une nation qui n'était plus seulement un territoire mais le lieu où les privilèges avaient été abolis ${ }^{10}$. Pour ce faire, ils devaient faire corps avec celle-ci, n'en être que le bras armé, non représenter un monde à part.

La loi fondatrice du 19 fructidor an VI

Il appartint aux néo-jacobins Jourdan et Delbrel, car le mérite de l'instauration de la loi revint tout autant à ce dernier, d'organiser ce que la Révolution avait ébauché. Jourdan revendiqua explicitement devant les Cinq-Cents la filiation révolutionnaire du projet en retraçant les étapes de son élaboration 
«La commission n'a pas modelé son projet sur des usages anciens à l'exemple des généraux français qui ont eu le courage de se soustraire à l'habitude pour vaincre et inventer une manière de combattre conforme au caractère national [On remarquera que, par une dérive insensible, ce sont les militaires et non les civils qui sont crédités de l'invention d'une armée nouvelle]. Elle a créé un mode de recrutement conforme aux principes de notre constitution. ${ }^{11}$

14

portaient la marque de l'urgence, ils imprimèrent un caractère durable et, surtout, posèrent les problèmes que soulevait l'existence de l'armée nationale en temps de paix. La commission qui élabora le projet travailla après la signature du traité de CampoFormio, elle pouvait envisager le long terme. Elle craignait la militarisation de la nation, comme les Constituants, et n'entendait pas la transformer en camp permanent. Elle acceptait en même temps le principe d'une armée encadrée par des professionnels devenus tels par goût du métier des armes ou parce qu'ils auraient contracté un engagement volontaire admis par la loi comme un des modes de recrutement de l'armée, à condition d'être gratuit. Mais il fallait éviter un double écueil la transformation des soldats et officiers de métier en caste particulière, le désintérêt de la nation qui s'en remettrait à un corps de métier du soin de sa défense parce qu'elle serait incapable de l'assurer par elle-même. Le meilleur remède était que, même en temps de paix, une fraction de la nation, composée de civils qui le redeviendraient une fois leur service accompli, amène au sein de la force armée un flux constamment renouvelé. En temps de guerre, ce serait de nouveau toute la nation, du moins ceux qui, parmi elle, seraient en âge et en état de combattre ${ }^{13}$, mais la loi laissait en suspens le problème de leur formation préalable. Quoi qu'il en soit, le caractère national d'une armée impliquait désormais que certains (pas nécessairement tous) lui consacrent une partie de leur temps sans songer à en faire une profession mais en leur qualité de citoyens.

II. Armée et citoyennetéL'invention de la citoyenneté et la figure du soldat-citoyen

Rétrospectivement, c'est la Révolution française qui nous semble avoir définitivement uni armée et citoyenneté ${ }^{14}$. Or ces liens n'allaient pas de soi comme le montra le débat à la Constituante mentionné plus haut. Plus précisément, les Constituants n'étaient prêts à accepter - dans une certaine mesure - que la seconde partie des vœux de Dubois-Crancé, c'est-à-dire que tout soldat demeure citoyen. Dans une certaine mesure, car l'Assemblée nationale fut prise au dépourvu devant les tensions entre soldats partisans du processus révolutionnaire et officiers nostalgiques de l'Ancien Régime et davantage encore devant les mutineries qui en résultèrent. Ces phénomènes prouvaient d'ailleurs la vanité des craintes des Constituants qui voyaient dans l'armée royale un pilier de l'absolutisme. C'est plutôt la limite entre exercice de la citoyenneté et politisation qui posa un problème. Certes, la loi du 28 février 1790 fit de tout soldat qui avait servi pendant seize ans un citoyen actif auquel il était possible de voter à son domicile celle du 5 août 1792 accorda cette qualité à tout combattant ayant accompli

Annales historiques de la Révolution française, 318 | octobre-décembre 1999 
une campagne ${ }^{15}$ mais, dès la Constituante, le ministère de la Guerre avait dû admettre la participation des soldats aux sociétés des Amis de la Constitution, sans qu'ils aient, toutefois, le droit de délibérer ${ }^{16}$.

Mais que tout citoyen devint soldat - première partie de la formule de Dubois-Crancé ne fut pas réalisé ni même accepté d'emblée. L'entrée en vigueur de ce principe procède d'un renversement de conception qui ne fut pas soudain. Il fallut la radicalisation de la situation politique autant que l'avènement d'une guerre nouvelle pour que le combat sur le champ de bataille fût perçu comme le prolongement de la lutte que devait mener le citoyen au sein de la cité, que le devoir de défense nationale fût maintenant considéré comme un attribut intrinsèque de la citoyenneté voire son essence même. Ce n'était donc plus seulement un devoir mais un droit, garantie de tous les autres.

La citoyenneté devenait aussi le fondement d'un instrument de combat de type nouveau qu'elle rendait par là même le plus efficace dans une guerre nouvelle. On pourrait aussi bien prétendre que c'est l'apparition de cette armée qui suscita le déploiement de la guerre des temps contemporains. En réfléchissant aux problèmes de tactique, les "militaires-philosophes " ${ }^{17}$ avaient inventé le citoyen sur le champ de bataille "idéal avant qu'il n'apparût dans la cité. Guibert qui souhaite que soit pleinement mise en œuvre la tactique nouvelle, esquissée par Frédéric II et correspondant selon lui à une guerre rationnelle ${ }^{18}$, appelle de ses vœux un soldat capable à la fois d'autonomie et d'obéissance, un «homme de la Raison» ${ }^{19}$. Cette conjugaison d'initiative et de discipline - la vertu, au sens antique du terme - c'est ce qu'on exige bientôt du citoyen qui participe à la vie politique de sa nation. Bientôt, le meilleur soldat paraîtra être celui qui se bat d'abord par conviction et on en arrivera même à assimiler celui qui lutte parce qu'il a des raisons profondes de le faire au seul citoyen, entendons celui qui n'en fait pas une profession. Le mythe superposera totalement le soldat-citoyen et le citoyen-soldat alors que Guibert n'envisageait pas que chaque citoyen combattît en personne ${ }^{20}$. Les Montagnards eux-mêmes n'acceptèrent cette conception que dans les circonstances extrêmes où les besoins en effectifs, jamais égalés, se conjuguaient avec la mise en œuvre de la nouvelle tactique.

Service militaire et citoyenneté

On a dit plus haut que la loi Jourdan, intégrant trois ans d'acquis révolutionnaires, se plaçait aussi dans la perspective d'une période de paix. Si la citoyenneté est la base de la force armée, cela n'implique pas qu'en temps ordinaire tous soient en permanence sous les armes. Les attendus de la loi affirment « Si les soldats de la patrie sont tous les Français en état de porter les armes, il faut déterminer comment appeler sous les drapeaux ceux dont la présence sera nécessaire... ${ }^{21}$, ce qui aura pour conséquence "...que beaucoup seront destinés à servir mais, vraisemblablement, peu serviront réellement ${ }^{22}$. La loi opère donc une distinction entre le devoir militaire, la conscription c'est-à-dire l'inscription sur les registres, devant laquelle tous sont égaux, et le service militaire qu'il n'est pas encore obligatoire d'accomplir en personne " ${ }^{23}$. Pour qu'une armée soit citoyenne, il suffit que le principe en soit proclamé, que tout Français soit, virtuellement, citoyen-soldat puisqu'il sait qu'il ne pourra être appelé que pour la défense de la liberté, celle de l'État au-dedans, celle de la République au-dehors, dit la loi ${ }^{24}$.

20 Ses auteurs sont très soucieux de la compatibilité de leur œuvre avec la liberté et l'égalité du citoyen avec la liberté d'abord, puisque, dans ce système de recrutement qui repose essentiellement sur les appels forcés, il importe d'établir des garde-fous. 
Non seulement le petit nombre des citoyens levés sera déterminé par un vote des assemblées, et on retrouve ici l'idée de primauté du pouvoir civil sur le pouvoir militaire, mais la durée qu'ils passeront sous les drapeaux sera limitée à cinq ans, une rotation des classes intervenant pour libérer chaque année les plus âgés. Avec l'égalité ensuite, car il faut trouver des critères de choix du contingent qui n'aient rien à voir avec la situation économique et sociale des conscrits ce sera l'âge, dit la loi puis, dans la pratique, dès la seconde levée directoriale, le tirage au sort. Le remplacement à prix d'argent est proscrit comme il l'était par le décret du 23 août. L'armée n'est pas encore, comme elle le sera sous la troisième République, le lieu de passage obligé de tout citoyen, sa seconde école et l'achèvement de son apprentissage civique. L'universalisation du service personnel n'apparaît pas comme la conséquence inéluctable de l'extension de la citoyenneté, voire l'antidote à la formation d'une caste militaire, le va-et-vient permanent souhaité entre le camp et la cité ne semble pas mieux assuré par la formation de gros bataillons.

21 Certes, la cité sera dans le camp parce que s'y opérera la jonction d'une minorité de civils avec des soldats de métier dont il est dit, dans la présentation du projet, qu'ils resteront citoyens « ...dès lors, il n'existera aucun motif de rivalité, de défiance, aucune ligne de démarcation entre le peuple et ses défenseurs " ${ }^{25}$. Mais les auteurs de la loi avouent leur impuissance à trancher sur les problèmes quand ils se posent en temps de guerre ils déclarent s'en remettre à la sagesse des assemblées pour fixer alors la durée du service. La loi reste muette également sur la relève des plus anciens par les plus jeunes dans ces circonstances. Le pouvoir civil demeure cependant maitre de déterminer le nombre des conscrits qui sera alors nécessairement plus élevé.

Du flou de la loi, Bonaparte allait tirer un parti magistral et la durée illimitée de la conscription ainsi que la formation des gros bataillons, dont le fondement théorique était la citoyenneté, ne servirent pas forcément la cause de la liberté au sein de la nation, encore moins au-dehors. Au point que bien des historiens estiment que l'armée napoléonienne, pourtant levée grâce à la loi Jourdan, redevint une armée de métier. On pourrait cependant objecter que les grognards gardèrent bien des traits des soldats de l'an II car ils se considéraient comme des citoyens en lutte contre toutes les formes et les «suppôts » du despotisme et étaient persuadés ainsi de libérer l'Europe ${ }^{26}$. C'est en s'appuyant sur ces convictions mythifiées que l'Empereur demanda et obtint longtemps de son armée ce que les monarques n'auraient pu ni même osé exigé de la leur. Génial manipulateur de l'héritage révolutionnaire, il comprit quelle force et quelle légitimité nouvelle, son instrument de guerre recevait du maintien de liens, mêmes dévoyés, avec la citoyenneté. Mais la captation de l'héritage n'avait-elle pas eu lieu dès brumaire lorsque les soldats crurent intervenir en tant que citoyens dans les affaires de la cité "Gardienne vigilante du legs révolutionnaire " comme le dit Jean-Paul Bertaud à propos du coup d'État du 18 fructidor ${ }^{27}$, l'armée finit par croire qu'elle incarnait seule l'idéal de citoyenneté et ses généraux plus encore. Peut-être était-ce l'effet pervers d'un processus que les Jacobins avaient déclenché et qui conduisit à une sorte de renversement des rôles c'est l'armée qui devint un modèle de citoyenneté pour la société et non plus l'inverse. Quand la loi Jourdan fut promulguée, bien des principes qu'elle proclamait avaient été en réalité mis à mal, le pouvoir militaire ne faisait plus corps avec le pouvoir civil, le Directoire s'était mis dans la main des généraux desquels seuls, aussi bien les révisionnistes que les néo-jacobins attendaient le salut politique.

III. Armée et sociétéÉtat-nation centralisateur et société un « dialogue » conflictuel 
Même si les conscrits maintenus indéfiniment au sein des troupes tendaient à se transformer en professionnels, l'armée napoléonienne ne pouvait totalement redevenir une armée de métier car, par le biais des levées conscriptionnelles, la société l'irriguait d'un sang neuf chaque année et, bientôt, plusieurs fois par an.

Tant s'en faut cependant que cette société se soit pliée docilement aux injonctions de la loi Jourdan. Celle-ci instaura un « dialogue » entre exigences de l'État et intérêts de la société, qui devait, dans certaines régions, demeurer conflictuel pendant presque un siècle. En fait, les trois premières levées qui se déroulèrent sous le Directoire mirent en lumière une France plurielle qui l'avait été face aux levées révolutionnaires. Il y a coïncidence, sinon superposition, entre la carte des comportements des Français devant les appels d'hommes de 1791 à 1793 et leurs attitudes devant la conscription. La nouvelle institution fit rejouer les conflits multiséculaires que l'État avait affrontés dans les «zones périphériques » de la France. Dans celles-ci, la loi Jourdan, conçue comme un instrument de l'unification nationale, la retarda ou plutôt révéla une très grande différence dans les degrés atteints par ce processus d'unification. On aurait tort d'imputer à la faiblesse du Directoire les difficultés que rencontra l'application initiale de la loi. En dépit de la propagande du nouveau régime, le Consulat et même l'Empire à ses débuts ne firent pas mieux si ce n'est pire.

Le facteur politique est loin d'être seul en cause ${ }^{28}$. La loi du 19 fructidor inaugure ainsi en certains lieux une lutte entre les représentants de l'État et la société civile ou plutôt elle la porte sur un nouveau terrain. Pourtant, après le 18 Brumaire, l'État se garde de renoncer à ses prérogatives on se souviendra que la tâche première assignée aux préfets est d'être des pourvoyeurs de la conscription. Il est en effet des départements où son acceptation est précoce voire immédiate ce sont ceux qui eurent la même attitude lors des appels de la Révolution ${ }^{29}$. D'autres, qui représentent la grande majorité, ne se signalèrent ni par des révoltes ni par un enthousiasme manifeste. Même dans les régions difficiles, l'État n'était pas partout aux prises avec de "petites Vendées ", les résultats des bons cantons, notamment les cantons urbains, compensèrent, numériquement parlant, ceux des cantons ruraux beaucoup plus rétifs ${ }^{30}$. Ainsi , au 18 Brumaire, le bilan n'était pas aussi négatif qu'une légende noire forgée après le coup d'État, le fit croire ${ }^{31}$.

Mais l'existence de ces régions moyennes ${ }^{32}$ ou bonnes ${ }^{33}$, eu égard au palmarès conscriptionnel, a été éclipsée par celle des zones de refus. L'étude des origines de ce refus a donné lieu à des analyses magistrales qui montrent que le tissu nécessaire à l'enracinement de la conscription n'existait pas sous le Directoire et n'existerait pas pendant longtemps ${ }^{34}$. Les facteurs de résistance, sociaux, économiques et culturels, en interaction les uns avec les autres, formaient un entrelacs difficile à dénouer et même à comprendre pour les représentants de l'État, en particulier les commissaires du Directoire enclins à déceler, dans toute émeute dirigée contre la conscription, le résultat d'un complot tramé par les aristocrates et les prêtres « fanatiques ». En réalité, ce complot, quand il y en avait un, bénéficiait d'un contexte défavorable à l'institution, il ne le créait pas. Distance, plus culturelle et linguistique que géographique, envers les centres de commandement de l'État-nation, isolement de populations vivant en autarcie, à tout le moins d'une agriculture fondée sur la petite propriété paysanne, se combinaient pour rendre incompréhensibles les exigences de l'État en dehors des périodes d'invasion - encore fallait-il avoir l'expérience de celle-ci. On a remarqué que le titre d'un des ouvrages mentionnés plus haut comporte les termes de "société 
civile ». La conscription n'avait pas réussi à transformer d'emblée, et elle ne devait pas le faire pendant longtemps, cette société civile en communauté de citoyens admettant le bien-fondé du devoir patriotique. Plus précisément, les conditions n'étaient pas réunies pour que la médiation du patriotisme produise son effet derrière les appels de la patrie, certains ruraux percevaient l'intrusion honnie de l'État.

La bonne volonté de certains autres manifeste au contraire qu'était intériorisé le contrôle étatique dont la conscription fut aussi un des instruments. En effet, l'addition des facteurs d'hostilité à la loi Jourdan dessine les traits d'un monde archaïque, l'adhésion à l'institution étant généralement signe d'entrée dans la modernité, bien que cet objectif n'ait pas été recherché délibérément par les partisans de la loi.

Formation d'une armée nationale et contraintes économiques

Parmi les nombreux éléments qui firent obstacle à l'application de la loi du 19 fructidor et qui avaient parfois rendu difficiles les levées de volontaires et la réquisition de février 1793, les structures économiques jouèrent un rôle déterminant. Les auteurs de la loi en étaient parfaitement conscients, ils l'étaient d'autant plus que, lorsque la loi fut votée, ils ne pouvaient que constater les progrès effrayants de la désertion qui expliquaient la diminution drastique des effectifs des armées du Directoire elles auraient perdu la moitié de leurs hommes de l'an II à l'an $\mathrm{V}^{35}$. Or, cette désertion était significativement une désertion à l'intérieur, preuve aux yeux de certains historiens ${ }^{36}$, de l'apatriotisme des ruraux qu'ils distinguent de l'antipatriotisme. Cette désertion n'est pas seulement due à la lassitude devant une guerre qui s'éternise et au cours de laquelle le fardeau pèse uniquement sur ceux qui tombaient sous le coup du décret du 23 août. Elle est liée au "poids terrible de la terre" ${ }^{37}$ dont les assemblées révolutionnaires n'avaient pris que peu à peu conscience pour un petit paysan privé de la main d'œuvre que représentent son ou ses fils, les réquisitions conduisent à la mort de son exploitation. C'est ainsi que la Convention montagnarde, pour faire admettre aux ruraux l'égalitarisme de la levée en masse, égalitarisme qui correspondait surtout aux vœux des citadins voire des salariés, avait développé une politique sociale en faveur des familles des défenseurs de la patrie, politique anticipatrice qui se poursuivit jusqu'à l'an $\mathrm{V}$ et qui eut aussi des retombées pour les soldats eux-mêmes, par le biais de pensions et d'une attention toute spéciale portée aux invalides et aux blessés ${ }^{38}$.

Les attendus de la loi du 19 fructidor sont très clairs sur ce problème.

«Tranquilles dans leurs foyers, les Français compris dans la conscription militaire ou, pour mieux dire, les défenseurs de la patrie, s'adonneront aux arts, au commerce et à l'agriculture. ॥ ${ }^{39}$

Des raisons idéologiques - évoquées plus haut - mais aussi économiques militaient pour que le mouvement qui portait les Français sous les drapeaux ne perturbe pas l'équilibre de la société. Si la commission « ...n'a voulu priver l'agriculture, les arts et le commerce que du nombre d'hommes absolument nécessaire à la défense de la patrie, c'est qu'elle n'entendait pas diviser la nation en deux classes, l'une militaire et l'autre civile ${ }^{40}$. Mais s'il était affirmé que le soldat - entendons l'appelé - devait retrouver son emploi après s'être acquitté de sa dette envers la patrie, exigence économique aussi bien qu'humaine ${ }^{41}$, la loi était muette quant à la reprise d'une politique en faveur des familles des conscrits. Il est vrai que cette politique avait répondu à un effort exceptionnel, même si elle avait survécu au démantèlement du système de l'an II, alors que les auteurs de la loi Jourdan - bien que néo-jacobins - se plaçaient dans la 
perspective du retour de la paix durant laquelle seul un petit nombre effectuerait son service pour un laps de temps limité.

En temps de guerre, les précautions prises pour assurer la compatibilité de l'intérêt général et du fonctionnement normal de l'économie, celle des intérêts particuliers et de l'égalitarisme, devaient se révéler caduques. Le remplacement, rétabli dès la seconde levée directoriale, en dépit de l'esprit de la loi et de ses modalités, laissait présager une pratique de l'institution qui finirait par la fausser, qui allait devenir, de toutes façons, le corollaire obligé du tirage au sort. Nous devons, cependant, nous garder de juger un tel phénomène à l'aune de nos mentalités contemporaines. Le remplacement qui avait existé pendant la levée des 300000 hommes et dès les levées de volontaires de l'été 1792, avant d'être prohibé pour la levée en masse, ne heurta pas l'égalitarisme des ruraux qui n'était point semblable au nôtre ni à celui des sans-culottes parisiens. Il paraissait plutôt une forme - de plus en plus dégradée et individualiste - du transfert de solidarité au sein même de la communauté villageoise ${ }^{42}$. Ainsi, si l'on ne peut abstraire la formation d'une armée de type nouveau des conditions économiques et sociales dans lesquelles elle s'insérait, il faut remarquer que les conséquences de la constitution de cette armée sont elles-mêmes un fait économique et social majeur.

C'est une armée nouvelle qui permit à Bonaparte de s'emparer du pouvoir puis de consolider sa domination que, grâce à elle, il étendit à une grande partie de l'Europe. En dépit des intentions initiales de ses créateurs, cette armée, par ses fondements même, donna une légitimité, nouvelle elle aussi et aux dimensions inégalées, à l'action de l'Empereur. Bien qu'il ait déformé plus que transformé l'héritage qu'il avait reçu des assemblées de la Révolution et du Directoire, ce type d'armée survécut aux "dangereuses formules » dans lesquelles Napoléon se lança ${ }^{43}$, au point de devenir un modèle pour l'Europe. Ses victoires même prouvaient son adéquation avec une nation et une société issues des temps nouveaux.

\section{NOTES}

1.Annie CRÉPIN, La conscription en débat ou le triple apprentissage de la nation, de la citoyenneté, de la République (1798-1889), Arras, Artois Presses Université, 1998, 256 p., p. 17.

2.Ce thème traversera le $\mathrm{xIX}^{\mathrm{e}}$ siècle jusqu'aux orléanistes conservateurs. Voir Annie CRÉPIN, De la nation-armée au service militaire obligatoire la conscription au XIXe siècle. Perspectives et méthodologie d'un champ historiographique. Dossier d'habilitation, Université d'Artois, 1999, $124 \mathrm{p}$.

3.Voir les travaux de Samuel ScotT dont The response of the Royal Army to the French Revolution, The role and development of the Line Army (1787-1793), Oxford, Clarendon Press, 1978, $244 \mathrm{p}$.

4.Jean-Paul BERTAUD, "Voies nouvelles pour l'histoire militaire, Annales historiques de la Révolution française, $\mathrm{n}^{\circ}$ 219, 1975, pp. 66-94. 
5.Alain EHRENBERG, Le corps combattant. Politique et pédagogie en démocratie, Paris, Aubier, 1983,213 p., p. 42

6.Je reprends pour la période qui commence en 1792 les remarques que Claude Nicolet a faites pour des périodes ultérieures “...guerres civiles - comme en 1848 et 1871 désastre ou crise militaire - comme en 1870, 1914 ou 1940 - lient toujours, par une fatalité contraignante, le sort de la République à celui des armes, la République à la patrie, même et surtout pour ceux qui croient de toute leur force au caractère universel de l'expérience française. La République se joue sous les yeux du genre humain. (Claude NICOLET, L'idée républicaine en France (1789-1924), Paris, Gallimard, 1982528 p., p. 112) .

7. Alain EHRENBERG, Le corps combattant..., op. cit., p.45.

8.Annie CRÉPIN, "Le Nord et le Pas-de-Calais face à la création de l'armée nationale, Revue du Nord, tome LXXV, n² 299, 1993, pp. 41-57..

9.Gustave VAlLÉE, La conscription dans le département de la Charente 1789-1807, Paris, Sirey, 1937, 708 p., p. 15.

10.Jean-Paul BERTAUD, La Révolution armée. Les soldats-citoyens et la Révolution française, Paris, Laffont, 1979, $380 \mathrm{p}$.

11.Arch. nat., C*I 120, Procès-verbaux du Conseil des Cinq-Cents, séance du 2 thermidor an VI.

12.Annie CRÉPIN, La conscription en débat..., op. cit., p. 25.

13.La troisième levée directoriale, celle du 10 messidor an VII, qui s'apparentait à une levée en masse, devait réaliser ce cas de figure.

14.La prégnance de cette conception jusqu'à nos jours peut expliquer pourquoi, en 1997, la conscription fut suspendue mais non supprimée, même si son maintien relève maintenant largement du domaine des principes.

15.Jean-Paul BERTAUD, Guerre et société en France de Louis XIV à Napoléon, Paris, 1998, 238

p., p. 34 .

16.Id., La Révolution armée..., op. cit., 238 p., p. 49.

17.Il est significatif que Servan, futur ministre girondin de la Guerre, intitule son ouvrage, paru en 1780 , Le soldat-citoyen.

18. Raymond MAS, "L'essai général de tactique de Guibert ou le rationalisme des Lumières face à la guerre, Colloque international de Clermont-Ferrand, 1985, La bataille, l'armée, la gloire, pp. 118-134.

19.Alain EHRENBERG, Le corps combattant..., op. cit., p.17 sq.

20.Alain Ehrenberg prétend, cependant, que son Essai général de tactique, paru en 1772, contient tout le discours révolutionnaire de la "nation en armes, mais on a vu plus haut qu'il avait fallu des circonstances exceptionnelles pour que ce discours entraînât la participation personnelle du citoyen encore ne s'entendit-elle pas comme la présence de tous sur le champ de bataille.

21.Arch. nat., C*I 120, Procès-verbaux..., op. cit., séance du 2 thermidor an VI.

22.Ibid., C I 439, Procès-verbaux..., op. cit., séances du 1er au 4 thermidor an VI.

23.Dans de toutes autres circonstances, la loi Gouvion-Saint-Cyr, refondatrice de la conscription, et la loi Soult reprendront cette distinction.

24.Arch. nat., C*I 120, Procès-verbaux..., op. cit., séance du 2 thermidor an VI.

25.Ibid.

26.Voir l'état de la controverse dans Annie CRÉPIN, Levées d'hommes et esprit public en Seine-et-Marne de la Révolution à la fin de l'Empire (1791-1815), Thèse, Université de Paris I, 1990, Livre I, p. 19 sq. 
27.Jean-Paul BERTAUD, La Révolution armée..., op. cit., p. 332.

28.Chacun des régimes du xix ${ }^{e}$ siècle devait se heurter aux mêmes difficultés dans les régions d'hostilité durable qui étaient parfois celles où il pensait compter - et où il comptait - ses meilleurs partisans même si la violence de cette résistance s'atténua peu à peu. Voir Maurice AGULHon, Histoire de la France rurale, Attitudes politiques, Paris, Éditions du Seuil, 1976,.560 p., tome III, pp.131-161.

29.Annie CRÉPIN, Levées d'hommes et esprit public..., op. cit.

30.Id., "Les communautés villageoises et la Grande Nation réticences et résistances face aux levées d'hommes et à la conscription, Colloque de Lille III, 1999, Du Directoire au Consulat le lien social et politique local dans la Grande nation, à paraître.

31.Isser Woloch, "Napoleonic conscription. State Power and civil society Past and Present, $\mathrm{n}^{\circ} 111,1986$, pp. 101-129 ; Id., The New Regime, Transformations of the French Civic Order, 1789-1820, New-York et Londres, Norton, 1994, 536 p.

32.L'une d'entre elles, la Charente, fut l'objet de l'œuvre maîtresse de Gustave VALLÉE, La conscription dans le département de la Charente 1798-1807, citée plus haut. De jeunes chercheurs ont continué à se pencher sur le cas de ces départements voir Kobo SEIGAN, La conscription dans le département de la Seine-Inférieure, du Directoire à la fin de l'Empire (an VI-1815), Thèse , Université de Paris I, 1998.

33.Annie CRÉPIN, Levées d'hommes et esprit public..., op. cit.

34.Jean WAQUET, "Réflexions sur les émotions populaires et le recrutement militaire de 1799 à 1831, Actes du 91e congrès national des Sociétés savantes, Rennes, 1966, pp. 51-74 ; "La société civile devant l'insoumission et la désertion à l'époque de la conscription militaire d'après la correspondance du ministère de l'Intérieur, Bibliothèque de l'École des Chartes, tome CXXVI, 1968, pp. 187-222 ; Louis BERGÈs, La société civile contre le recrutement à l'époque de la conscription militaire (1798-1814), le cas des départements aquitains, Thèse, École des Chartes, Paris, 1987 ; Bruno CIOTTI, Du volontaire au conscrit, les levées militaires dans le département du Puy-de-Dôme sous la Révolution (1791-an VII), Thèse, Université de Lyon II, 1995-1997.

35.Jean-Paul BERTAUD, La Révolution armée..., op. cit., p. 271.

36.Georges SANGNIER, La désertion dans le Pas-de-Calais de 1792 à 1802, Doullens, Dessaint, 1965, 267 pp. ; Robert Legrand, Aspects de la Révolution en Picardie, Abbeville, Lafosse, 1957, 63 p.

37.Jean-Paul BERTAUD, Débat à propos de la communication d'Allan Forrest, “La formation des attitudes villageoises devant le service militaire (1791-1814), Colloque international de Clermont-Ferrand, 1985, La bataille, l'armée, la gloire, pp. 193-197. 38.Isser Woloch, The French Veteran, from the Revolution to the Restoration, Chapel Hill, University of North Carolina Press, 1979, 392 pp. ; "War-widows pensions social policy in revolutionary and napoleonic France, Societas, Volume VI, $n^{\circ} 4,1976$.

39.Arch. nat., C I 439, Procès-verbaux... séances du 1er au 4 thermidor an VI.

40.Ibid., C*I 120, Procès-verbaux..., séance du 2 thermidor an VI.

41.Ibid., C I 439, Procès-verbaux..., séances du 1er au 4 thermidor an VI.

42.Annie CRÉPIN, Levées d'hommes et esprit public..., op. cit.

43.Jean JAURÈs, L'armée nouvelle, Paris, Éditions sociales, réédition 1978, 408 p., p. 45. 


\section{RÉSUMÉS}

Le 18 Brumaire, Bonaparte trouva un instrument de guerre entièrement forgé par la Révolution et le Directoire. Il devait de plus en plus s'éloigner des intentions initiales des créateurs d'une armée nouvelle. Celle-ci, pourtant, survécut au détournement de l'héritage que Bonaparte avait reçu en la matière tant elle était fondée sur des rapports nouveaux avec une nation et une société, elles aussi nouvelles, tant elle était en adéquation avec l'invention de la citoyenneté.

18 Brumaire, a Milestone in the Creation of a New Army. On 18 Brumaire, Bonaparte inherited an instrument of war entirely forged by the Revolution and the Directory. He was effectively to deviate from the intentions of the creators of the new Army. Nevertheless, the Army managed to survive the misappropriation of the legacy Bonaparte had received, such was the resilience of the new bonds which linked it to a nation and a society themselves forged anew, and so closely did it find itself in harmony with the invention of citizenship.

Il 18 brumario, una tappa nella costituzione d'un esercito nuovo. I1 18 brumario, Bonaparte trovò uno strumento di guerra interamente forgiato dalla Rivoluzione e dal Direttorio. Esso doveva sempre più allontanarsi dalle intenzioni iniziali dei creatori di un esercito nuovo. Questo, tuttavia, sopravvisse al dirottamento dell'eridità che Bonaparte aveva recivuto in tale materia per il fatto di essere fondato su rapporti nuovi tra una nazione ed una società anch'esse nuove e per il suo adeguamento con l'invenzione della cittadinanza.

El 18 de brumario, tiene Bonaparte un instrumento de guerra que era un producto de la Revolución y del Directorio. Sin embargo, este sebrevivió a la corrupción de la herencia que recibió Bonaparte porque estaba fundado este ejército sobre nuevas relaciones entre una nación y una sociedad ellas también nuevas, y porque estaba en adecuación con la invención de la ciudadanía.

Der 18. Brumaire, eine neue Stufe im Aufbau eines neuen Heeres. Am 18. Brumaire fand Bonaparte ein von der Revolution und dem Direktorium neugeschaffenes Kriegswerkzeug. Er entfernte sich immer mehr von den ursprünglichen Absichten der Schöpfer dieses neuen Heeres. Dieses überlebte jedoch dieser Hinterziehung der Erbschaft, weil es auf neuen Beziehungen zwischen der Nation und einem auch neuen Gesellschaftssystem gegründet war und weil es der Erfindung der Staatsbürgerschaft vollkommen entsprach.

\section{AUTEUR}

\section{ANNIE CRÉPIN}

Université d'Artois 